\title{
Comorbilidad en una Muestra de Pacientes con Agorafobia: La Presencia de la Ideación Suicida
}

\author{
Comorbidity in a Sample of Patients with Agoraphobia: The Presence of Suicidal \\ Ideation
}

\author{
Wenceslao Peñate ${ }^{1}$, Laura Dorta-Concepción ${ }^{2}$, Yolanda Álvarez-Pérez ${ }^{3}$, Carmen T. Pitti ${ }^{4}$, \\ Mary Luz Villaverde ${ }^{5}$ y Juan Manuel Bethencourt ${ }^{6}$
}

\begin{abstract}
Resumen
La agorafobia representa uno de los trastornos mentales más incapacitantes. Es una fobia con un alto nivel de comorbilidad, especialmente con otros trastornos de ansiedad y con trastornos afectivos. De manera más grave, algunos pacientes presentan ideación suicida. Con el este trabajo se pretendió conocer la comorbilidad de la agorafobia en una muestra de 254 pacientes, especialmente en relación con la ideación suicida. Los resultados indicaron un alto porcentaje de pacientes con depresión y ansiedad social. La presencia de la ideación suicida alcanzó a un tercio de la muestra. Esta ideación estuvo especialmente relacionada con el nivel de depresión. La presencia de crisis de pánico, ansiedad social y consumo de sustancias no mejoró significativamente la predicción de la ideación suicida en personas con agorafobia. Estos resultados se discuten a la relevancia de la comorbilidad en el suicidio y sus implicaciones en la evolución de la agorafobia.
\end{abstract}

Palabras clave: agorafobia, comorbilidad, depresión, ideación suicida

\begin{abstract}
Agoraphobia represents one of the most disabling mental disorders. Comorbidity is very frequent, especially with other anxiety disorders and with affective disorders. Especially relevant is the fact that several patients have suicidal thoughts. In severe levels, some patients can present suicidal ideation. The aim of this study was to know the comorbidity rates in a sample of 254 patients with agoraphobia, particularly related with depression and suicidal ideation. Results showed a high proportion of patients with depression and with social anxiety. Suicidal thoughts were found in one third of patients. These thoughts were especially related to level of depression. The presence of panic attacks, social anxiety and substance abuse did not significantly improve the prediction of suicidal ideation in people with agoraphobia. These results are discussed according to the relevance of comorbidity in suicidal behavior, and its implications in the evolution of agoraphobia.
\end{abstract}

Keywords: agoraphobia, comorbidity, depression, suicidal ideation

Agradecimientos: Este estudio ha sido financiado por los proyectos de investigación PSI 2013-42912-R y PSI2017-83222-R del Ministerio de Economía y Competitividad del Gobierno de España.

\footnotetext{
${ }^{1}$ Doctor en Psicología. Universidad de la Laguna. Dto. Psicología Clínica, Psicobiologia y Metodología. Apdo 456, Campus de Guajara 38200 La Laguna. Santa Cruz de Tenerife, España. Tel.: +34922317485. Correo: wpenate@ull.edu.es

${ }^{2}$ Máster en Psicología Clínica y de la Salud. Centro de Psicología y Logopedia Halocen. La Laguna, Santa Cruz de Tenerife, España.

${ }^{3}$ Doctora en Psicología. Servicio de Evaluación y Planificación. Servicio Canario de la Salud. Gobierno de Canarias. Santa Cruz de Tenerife, España.

${ }^{4}$ Doctora en Psicología. Dirección General de Salud Pública. Servicio Canario de la Salud. Gobierno de Canarias. Santa Cruz de Tenerife, España.

${ }^{5}$ Doctora en Medicina y Cirugía. Universidad de La Laguna. Dto. Medicina Interna, Dermatología y Psiquiatría. La Laguna, Santa Cruz de Tenerife, España.

${ }^{6}$ Doctor en Psicología, Universidad de la Laguna, Dto. Psicología Clínica, Psicobiologia y Metodología. La Laguna, Santa Cruz de Tenerife, España.
}

Revista Iberoamericana de Diagnóstico y Evaluación - e Avaliação Psicológica. RIDEP · No55 · Vol.2 · 173-183 · 2020

ISSN: 1135-3848 print /2183-6051online 


\section{Introducción}

La agorafobia está considerada la fobia más grave y la más incapacitante, pues afecta a múltiples dominios de la vida de la persona. Las clasificaciones psicopatológicas (American Psychiatric Associaton, APA, 2013; World Health Organization,WHO, 1992) incluyen a la agorafobia en los trastornos de ansiedad y la definen como el miedo a estar en lugares o situaciones de los cuales pueda ser difícil o embarazoso escapar o en los cuales pueda no disponerse de ayuda en el caso de tener un ataque de pánico o síntomas similares. Como consecuencia de este miedo, la persona evita las situaciones temidas, o las soporta con gran ansiedad. Puede diagnosticarse con o sin ataques de pánico. Tiene un comienzo relativamente temprano (15-17 años), con una prevalencia a lo largo de la vida de 2,5\% de la población, siendo significativamente más frecuente en mujeres (3,2\% de prevalencia) (Kessler, Petukhova, Sampson, Zaslavsky, \& Wittchen, 2012).

La agorafobia es altamente comorbida. Es bien conocida su relación con la ansiedad social (Magee, Eaton, Wittchen, McGonagle, \& Kessler, 1996), como un elemento de vulnerabilidad para la inestabilidad emocional y las dificultades interpersonales (Delgado, Inglés, Aparisi, GarcíaFernández, \& Martínez-Monteagudo, 2018). Brown, Campbell, Lehman, Grisham y Mancill (2001), en una muestra de 324 personas con agorafobia, encontraron que el $60 \%$ recibieron un diagnóstico adicional con otro trastorno de ansiedad, y el $47 \%$ con un trastorno depresivo. Además, observaron que se daba una menor comorbilidad cuando la agorafobia cursaba sin trastorno de pánico.

En relación con otro tipo de trastornos, se han encontrado niveles apreciables de comorbilidad con conductas adictivas. Los pacientes con trastorno de pánico con o sin agorafobia suelen ser comórbidos con el abuso del alcohol y el abuso de sustancias, y los que coexisten con el abuso de sustancias son más propensos a tener conductas suicidas (Huang, Yen, \& Lung, 2010). En este sentido, se ha podido constatar que las personas con agorafobia poseen un apreciable riesgo suicida. Starcevic, Bogojevic, Marinkovic y Kelin (1999), observaron una prevalencia de riesgo suicida en uno de cada tres pacientes. Tasas similares se encontraron en estudios posteriores (An et al., 2018; Grant et al., 2003; Inoue, Kaiya, Hara, \& Okazaki, 2016), siendo, con el trastorno por estrés postraumático, la patología ansiosa más vinculada al pensamiento y al intento suicida.

A nivel general, se ha observado que la comorbilidad de la agorafobia con otros trastornos de ansiedad y con trastornos depresivos parece un elemento clave para ese riesgo suicida (Teismann et al., 2018), incluyendo a ese riesgo en la población juvenil (Gómez-Tabares, AgudeloOsorio, \& Grisales-Aguirre, 2020), aunque, de acuerdo con el estudio de Orri et al., (2018), en el caso de adolescentes, esa comorbilidad no es suficiente $y$ es necesario la presencia de irritabilidad. Los datos más sólidos para explicar las tendencias suicidas en personas con agorafobia se encuentran en su comorbilidad con los procesos depresivos, considerándose esta relación la de mayor riesgo (Baca \& Aroca, 2014). Sareen, Cox y Afifi (2005), encontraron que las personas con trastornos de ansiedad comórbidos con trastornos del estado de ánimo presentaban un mayor riesgo de intentos de suicidio que las personas con sólo trastornos del estado de ánimo. Starcevic et al., (1999), encontraron que alrededor del $80 \%$ de los pacientes con pensamientos suicidas también cumplían los criterios de algún trastorno afectivo, especialmente depresión mayor. Datos similares sobre el papel de la depresión fueron encontrados por Sánchez-Álvarez, de la Torre-López y PérezCostillas (2018). Norton, Temple y Pettit (2008), argumentaron que este aumento de intentos de suicidio en este tipo de población puede ser debido a la afectación de la vida funcional, social y laboral que conlleva la agorafobia. No obstante, Ashrafioun, Pigeon, Conner, Leong y Oslin (2016), encontraron que esa relación entre agorafobia y depresión estaba más vinculada con la ideación que con los intentos suicidas.

Sin embargo, además de la comorbilidad con los trastornos afectivos, también ha podido observarse la presencia de la ideación suicida en personas con agorafobia con otro tipo de variables, como el estrés postraumático, abuso de alcohol/fármacos/drogas, trastorno de personalidad límite o ruptura de parejas (Bados, 2006). En este sentido, en el trabajo de Miché et al. (2017), la relación más estrecha fue entre 
suicidio y estrés postraumático, pero esa relación era mayor en la medida en que se incrementaba la comorbilidad (como la presencia de depresión y abuso de sustancias). En la citada investigación de Sareen et al., (2005), también encontraron una relación significativa entre agorafobia, trastorno de pánico e intentos de suicidio. En la revisión de Kilbane, Gokbayrak, Galynker, Cohen y Tross (2009), se constató que la comorbilidad entre los trastornos de ansiedad (especialmente con pánico) posee un mayor peso explicativo de la ideación suicida, en comparación con la comorbilidad con los trastornos depresivos. En un estudio de moderadores y mediadores entre el pánico, síntomas de agorafobia y la ideación suicida (Huang et al., 2010), se encontró que los síntomas de pánico más graves, un bajo apoyo social, edades jóvenes y el consumo actual de alcohol, estaban relacionados con ideas de suicidio.

Sin embargo, esa relación entre comorbilidad entre trastornos de ansiedad y suicidio no siempre se ha podido verificar. El meta-análisis llevado por Bentley et al. (2016), la relación entre trastornos de ansiedad y suicidio fue más débil y prácticamente sólo se observó esa relación para el trastorno de estrés postraumático y sólo para la ideación suicida (no para intentos suicidas). En el estudio de Abreu et al. (2018), los trastornos de ansiedad no añadieron capacidad predictiva a la ideación suicida. En el citado estudio de Ashrafioun et al. (2016), sólo el consumo de drogas (ilícitas) se relacionó significativamente con los intentos suicidas.

De acuerdo con estos datos, la gravedad de la agorafobia se ve incrementada por la elevada comorbilidad que posee con otros trastornos. Un aspecto crítico es la comorbilidad de la agorafobia con la presencia de ideación o planificación suicida y su vinculación con la depresión, el pánico o conductas adictivas. Sin embargo, los datos no son concluyentes. En algunos estudios los pensamientos suicidas se han asociado a la comorbilidad con depresión, pero en otros se ha asociado a la comorbilidad con otros trastornos de ansiedad o el abuso de sustancias. Además, algunos estudios parecen indicar que, a mayor presencia de comorbilidad, mayor riesgo suicida.

En este sentido, este trabajo tiene como objetivo general analizar esa comorbilidad en una muestra de personas con agorafobia. En concreto con (i) la presencia de trastornos de pánico, fobia social, depresión, conductas adictivas e ideación suicida. Además, (ii) se analizará la relación de la comorbilidad con la ideación suicida y (iii) en qué medida se incrementa el riesgo suicida a mayor comorbilidad.

\section{Método}

\section{Participantes}

La muestra final estuvo compuesta por 254 personas con diagnóstico de agorafobia. Los participantes fueron derivados desde las Unidades de Salud Mental Comunitaria y desde los centros de Atención Primaria, al Servicio de Psiquiatría del Hospital Universitario de Canarias. Se incluyeron aquellos que cumplían con los criterios de la CIE-10 (WHO, 1992) para el diagnóstico de la agorafobia (con/sin pánico). Los criterios de exclusión fueron: personas diagnosticadas con psicosis, trastornos de personalidad y otros trastornos de ansiedad donde el trastorno de agorafobia era un diagnóstico secundario. Del total de la muestra, 147 (59\%) cumplieron los criterios de agorafobia con pánico.

La media de edad de la muestra fue de 38.64 años (desviación típica, 10.03). El rango osciló entre 20 y 61 años. La mayoría de los participantes eran mujeres $(72.8 \%)$. En cuanto al estado civil, el $43.1 \%$ eran solteros, $44.4 \%$ casados, y el $12.5 \%$, divorciados o separados. En cuanto al nivel de estudios, $19.3 \%$ tenían educación primaria, $60.6 \%$ tenían educación secundaria, y $20 \%$ tenían un título universitario. La cronicidad de los síntomas clínicos fue de 1 a 45 años, con un promedio de 9.62 años. Con respecto a esa evolución, en el $22.8 \%$ de los casos había debutado con el trastorno hacia 1-2 años, en el $18.7 \%$, de 3- 5 años y en el $58.5 \%$ de los casos fue de 6 años o más.

Todos los participantes firmaron un formulario de consentimiento, aprobado por el comité de ética institucional del Hospital Universitario de Canarias (HUC).

\section{Instrumentos}

Se utilizaron dos entrevistas: (i) una entrevista inicial para recoger datos socio-demográficos (género, edad, nivel de estudios, estado civil, 
situación laboral y cronicidad), la identificación de consumo de sustancias y la presencia de ideación suicida. (ii) La entrevista estructurada CIDI 2.1 (Kessler \& Üstün, 2004) en sus apartados referidos a agorafobia, pánico, y otras fobias.

Inventario de Agorafobia (AGF, Echeburúa, Corral, García, Páez, \& Borda, 1992). El inventario AGF está compuesto por 69 ítems que se contestan en una escala tipo Likert. Consta de tres subescalas: motora, psicofisiológica y cognitiva. La subescala motora contiene posibles situaciones temidas. Cada ítem se responde dos veces, de acuerdo a cómo se comporta solo y cómo lo hace en compañía. La subescala psicofisiológica contiene las sensaciones corporales que le preocupan cuando está ante una situación temida. La subescala cognitiva contiene ítems sobre los pensamientos en relación con la vivencia de una crisis de ansiedad. Los puntos de corte se establecen en 176 para el inventario global y de 96, 61 y 30 en la escala de respuestas motoras, psicofisiológicas y cognitivas, respectivamente. En el trabajo original, se informa de buenas propiedades psicométricas en la detección de la agorafobia y en la selección de conductas-problemas para el tratamiento (Echeburúa et al., 1992). Este instrumento se utilizó, conjuntamente con la CIDI, para corroborar el diagnóstico de agorafobia de los participantes.

Liebowitz Social Anxiety Scale (LSAS, Liebowitz, 1987). La LSAS es una escala diseñada para evaluar la gravedad del trastorno de ansiedad social. La escala consta de 24 ítems referidos a 24 situaciones, que se responde en dos ocasiones: (i) temor experimentado y (ii) grado de evitación de esas situaciones. Las respuestas se califican mediante una escala Likert de 4 puntos, de 0 (nada) a 3 (mucho). En sumatorio de las respuestas permite obtener una puntuación general en ansiedad social (leve, moderada o grave). Se obtuvieron altos coeficientes de consistencia interna (.92 para LSAS-miedo, 92 para LSASevitación y .96 en la puntuación total) y una adecuada sensibilidad a los cambios producidos por el tratamiento (Heimberg et al., 1999).

El Inventario de Depresión de Beck, edición revisada (BDI-II; Beck, Steer, \& Brown, 1996). El BDI-II Es un instrumento autoadministrado de 21 ítems que mide la severidad de la depresión en adultos y adolescentes mayores de 13 años. Cada ítem se califica en una escala de 4 puntos que va desde el 0 hasta el 3. Los ítems referidos a problemas de apetito y sueño poseen 7 alternativas. El inventario permite clasificar a los evaluados en cuatro categorías: no depresión, leve, moderada y grave. Los autores informaron de adecuados niveles de consistencia interna, validez criterial y discriminante (Beck et al., 1996).

\section{Procedimiento}

Desde las unidades de salud mental y desde atención primaria las personas con agorafobia fueron derivadas a la Unidad de Investigación del HUC, dentro de un programa específico ofertado para el tratamiento combinado psicoterapia / psicofármacos para la agorafobia, que se llevó a cabo entre 2010 y 2016. Una vez que acudían al servicio, se les verificaba el diagnóstico empleando la entrevista CIDI 2.1 (Kessler \& Üstün, 2004) y el Inventario de AGF (Echeburúa et al., 1992). Comprobado el diagnóstico, se les administraba el resto de las pruebas.

\section{Análisis de datos}

Se realizaron análisis de frecuencias en las diferentes variables y categorías diagnósticas. Los contrastes entre categorías se llevaron a cabo mediante $\mathrm{X}^{2}$. Para los contrastes de puntuaciones medias entre personas con ideación suicida y sin ideación, se aplicaron ANOVAs. La contribución relativa de las distintas variables posicopatológicas a la ideación suicida se analizó mediante regresión múltiple. Finalmente, para conocer la asociación de todas las variables medidas con la ideación suicida se llevó a cabo una regresión logística. Se creó una variable sobre el consumo de sustancias, dando un valor de 1.00 a sí consumían alcohol, tabaco, cannabis, estimulantes $\mathrm{u}$ otros, pudiéndose obtener una puntuación máxima de 5.0.

\section{Resultados}

Los datos iniciales en el inventario AGF confirmaron la situación clínica de los pacientes evaluados. Globalmente, se obtuvo una puntuación media (M) de 298.23 (punto de corte a partir de 176), y una desviación típica (DT) de 
Tabla 1. Distribución de frecuencias (porcentajes) de la presencia de depresión, ansiedad y ideación suicida en una muestra de personas con agorafobia con pánico y sin pánico

\begin{tabular}{lcccccc}
\hline & Depresión & $\begin{array}{c}\text { Ansiedad } \\
\text { social }\end{array}$ & $\begin{array}{c}\text { Pensamiento } \\
\text { suicida }\end{array}$ & & & \\
\hline \\
Agorafobia sin pánico & SÍ & NO & SÍ & NO & SÍ & NO \\
Agorafobia con pánico & $88.4 \%$ & $11.6 \%$ & $76.8 \%$ & $23.2 \%$ & $65.3 \%$ & $34.7 \%$ \\
Total & $92.9 \%$ & $7.1 \%$ & $80.9 \%$ & $19.1 \%$ & $61.6 \%$ & $38.4 \%$ \\
Chi cuadrado & $91.1 \%$ & $8.9 \%$ & $78.2 \%$ & $21.8 \%$ & $63.1 \%$ & $36.9 \%$ \\
\hline
\end{tabular}

\section{Adicciones}

\begin{tabular}{lccccccccccc} 
& \multicolumn{2}{c}{ Tabaco } & \multicolumn{2}{c}{ Alcohol } & \multicolumn{2}{c}{ Cannabis } & \multicolumn{2}{c}{ Estimulantes } & \multicolumn{2}{c}{ Otros } \\
\cline { 2 - 11 } & \multicolumn{2}{c}{ SÍ } & NO & SÍ & NO & SÍ & NO & SÍ & NO & SÍ & NO \\
\cline { 2 - 12 } $\begin{array}{l}\text { Agorafobia } \\
\text { sin pánico }\end{array}$ & $24.5 \%$ & $75.5 \%$ & $3.9 \%$ & $96.1 \%$ & $2.9 \%$ & $97.1 \%$ & $2.9 \%$ & $97.1 \%$ & $6.9 \%$ & $93.1 \%$ \\
$\begin{array}{l}\text { Agorafobia } \\
\text { con pánico }\end{array}$ & $28.6 \%$ & $71.4 \%$ & $6.8 \%$ & $93.2 \%$ & $3.4 \%$ & $96.6 \%$ & $0.0 \%$ & $100.0 \%$ & $1.4 \%$ & $98.6 \%$ \\
$\begin{array}{l}\text { Total } \\
\text { Chi cuadrado }\end{array}$ & $26.9 \%$ & $73.1 \%$ & $5.6 \%$ & $94.4 \%$ & $3.2 \%$ & $96.8 \%$ & $1.2 \%$ & $98.8 \%$ & $96.4 \%$ & $3.6 \%$ \\
\hline
\end{tabular}
Nota. $* p \leq .05$

Tabla 2. ANOVA sobre las puntuaciones en agorafobia, depresión, ansiedad social y consumo de sustancias, de acuerdo a sí habían tenido ideación/intentos suicidas $(\mathrm{N}=80)$ o no $(\mathrm{N}=141)$

\begin{tabular}{lcccccc}
\hline $\begin{array}{l}\text { Variables } \\
\text { dependientes }\end{array}$ & Ideación suicida & $M$ & $D T$ & $F$ & $p$ & $n^{2}$ \\
\hline Agorafobia & NO & 45.44 & 14.15 & 12.12 & .001 & .052 \\
& SÍ & 53.02 & 17.79 & & & \\
Depresión & NO & 22.86 & 12.22 & 49.34 & .000 & .184 \\
& SÍ & 34.93 & 12.36 & & & \\
Fobia social & NO & 52.65 & 27.52 & 15.22 & .000 & .065 \\
Consumo de & SÍ & 69.89 & 37.68 & & &. \\
sustancias & NO & .38 & .68 & .624 & .430 & .003 \\
\hline
\end{tabular}

Nota. $\mathrm{M}=$ Puntuación media; DT=desviación típica; $\mathrm{F}=\mathrm{F}$ de Fisher; $p=$ probabilidad; $\mathrm{n}^{2}=$ eta cuadrado

93.22; para la subsección de comportamiento motor M=143.67 (punto de corte de 96), $\mathrm{DT}=60.25$; subsección fisiológica $\mathrm{M}=107.89$, DT=33.67 (punto de corte de 61); y para la subsección cognitiva $\mathrm{M}=46.67$ (punto de corte de 30), DT=19.30.

Con respecto al primer objetivo, para el análisis de comorbilidad se llevó a cabo un análisis descriptivo sobre la presencia de sintomatología depresiva, de ansiedad social y de pensamientos suicidas en la muestra de personas con agorafobia. La muestra se dividió en personas con agorafobia con o sin trastorno de pánico. Para el caso de depresión y ansiedad, se agruparon los datos en NO presencia del trastorno y Sí presencia del trastorno (para ello se agruparon las categorías de leve, moderado o grave). Para el caso de los pensamientos suicidas, se agruparon la ideación y los intentos. En la Tabla 1 se resumen los datos.
Como puede observarse, en general, la presencia de depresión, ansiedad y pensamientos suicidas es muy elevada, destacando los procesos depresivos que se encuentran en nueve de cada 10 pacientes con agorafobia. En el caso de la ansiedad social, ésta se encuentra en tres de cada cuatro casos. Llamativamente, la ideación suicida está presente en casi dos de cada tres casos de agorafobia. Analizando, según estuviera presente un trastorno de pánico o no, no se observaron diferencias significativas, ya que ningún contraste de chi cuadrado alcanzó significación estadística.

Con respecto a las conductas adictivas y el consumo frecuente de sustancias, los niveles de comorbilidad se invierten y son muy escasos. El consumo de tabaco es el más frecuente, alcanzando aproximadamente a una de cada cuatro personas con agorafobia. De nuevo, analizando por separado los porcentajes por la 
Tabla 3. Regresión múltiple (método directo) sobre la ideación suicida en una muestra de personas con agorafobia $(n=254)$, utilizando como predictores la puntuación en agorafobia, en depresión, en fobia social y en consumo de sustancias

\begin{tabular}{lccccc}
\hline Variables & $B$ & $E . T$. & $B$ tipificada & $t$ & $p$ \\
\hline (Constante) & -.17 & .11 & & -1.54 & .125 \\
Agorafobia & -.00 & .00 & -.05 & -.65 & .518 \\
Depresión & $\mathbf{. 0 2}$ & $\mathbf{. 0 0}$ &. $\mathbf{4 4}$ & $\mathbf{5 . 8 5}$ & $\mathbf{. 0 0 0}$ \\
Fobia social & .00 & .00 & .14 & 1.96 & .052 \\
Consumo de sustancias & .04 & .05 & .05 & .86 & .393 \\
\hline
\end{tabular}

Nota . B=Beta; E.T.=error típico; $\mathrm{t}=\mathrm{t}$ de student; $p=$ probabilidad

Tabla 4. Regresión logística (pasos sucesivos) sobre ausencia/presencia de ideación suicida. tomando como variables predictoras las variables psicológicas comórbidas y variables socio-demográficas

\begin{tabular}{lcccccccc}
\hline & $B$ & $E . T$. & Wald & $g l$ & $p$ & Exp (B) & \multicolumn{2}{c}{$I C$} \\
\hline EDAD & $\mathbf{. 2 5}$ & $\mathbf{. 0 9}$ & $\mathbf{6 . 1 9}$ & $\mathbf{1}$ & $\mathbf{. 0 1 3}$ & $\mathbf{. 7 8}$ & $\mathbf{. 7 8}$ & $\mathbf{. 6 4}$ \\
NE & & & 7.08 & 4 & .132 & & & $\mathbf{1 8 . 1 0}$ \\
Primarios & $\mathbf{1 . 5 0}$ & $\mathbf{. 7 1}$ & $\mathbf{4 . 4 9}$ & $\mathbf{1}$ & $\mathbf{. 0 3 4}$ & $\mathbf{4 . 5 0}$ & $\mathbf{1 . 1 2}$ & $\mathbf{1 8 . 1 0}$ \\
Secundarios & $\mathbf{1 . 3 8}$ & $\mathbf{. 6 4}$ & $\mathbf{4 . 6 7}$ & $\mathbf{1}$ & $\mathbf{. 0 3 1}$ & $\mathbf{3 . 9 9}$ & $\mathbf{1 . 1 4}$ & $\mathbf{1 4 . 0 2}$ \\
Titulado medio & .62 & .68 & .83 & 1 & .361 & 1.85 & .49 & 6.95 \\
Licenciado & .91 & .76 & 1.43 & 1 & .232 & 2.48 & .56 & 10.96 \\
SL & & & 7.77 & 2 & .021 & & & \\
Incapacidad & $\mathbf{1 . 6 3}$ & $\mathbf{. 6 1}$ & $\mathbf{7 . 1 9}$ & $\mathbf{1}$ & $\mathbf{. 0 0 7}$ & $\mathbf{5 . 0 9}$ & $\mathbf{1 . 5 5}$ & $\mathbf{1 6 . 7 4}$ \\
Paro & $\mathbf{1 . 5 3}$ & $\mathbf{. 5 9}$ & $\mathbf{6 . 7 7}$ & $\mathbf{1}$ & $\mathbf{. 0 0 9}$ & $\mathbf{4 . 6 2}$ & $\mathbf{1 . 4 6}$ & $\mathbf{1 4 . 6 5}$ \\
Depresión & $\mathbf{. 0 9}$ & $\mathbf{. 0 2}$ & $\mathbf{2 8 . 6 3}$ & $\mathbf{1}$ & $\mathbf{. 0 0 0}$ & $\mathbf{1 . 0 9}$ & $\mathbf{1 . 0 6}$ & $\mathbf{1 . 1 2}$ \\
Constante & -5.91 & 1.65 & 12.81 & 1 & .000 & .00 & & \\
\hline
\end{tabular}

Nota. IC=intervalo de confianza; B=Beta; ET=error típico; Wald=coeficiente Wald; gl=grados de libertad; p=probabilidad estadística; $\operatorname{Exp}(\mathrm{B})=$ odds ratio; $\mathrm{EC}=$ estado civil; $\mathrm{NE}=$ nivel de estudios; $\mathrm{SL}=$ situación laboral.

presencia o no de pánico, sólo se observan resultados significativos para el consumo de estimulantes y otro tipo de drogas, siendo más frecuente su consumo sin pánico que con pánico, pero con niveles de incidencia realmente bajos.

Con respecto al segundo objetivo, la presencia de la ideación suicida, se quiso conocer cómo ésta se relacionaba con los niveles de gravedad de la agorafobia (puntuación total en el cuestionario AGF), de la depresión, la ansiedad social y el consumo de sustancias. La variable suicidio se dividió en dos categorías: no ideación ni intentos (NO) y sí intentos o ideación (SÍ). Con esta variable se llevó a cabo un ANOVA sobre las variables psicopatológicas citadas como variables dependientes. Se tomó como covariable si la agorafobia cursaba o no con ataques de pánico. En la Tabla 2 se resume los datos obtenidos.

Como puede observarse, el grupo de personas con agorafobia y presencia de ideación / intento suicidas obtuvieron una puntuación significativamente más alta en agorafobia, en depresión y en ansiedad social. En el caso de la depresión se obtuvo un tamaño del efecto (eta cuadrado) grande. Sin embargo, el consumo de sustancias no alcanzó significación estadística. La presencia o no de pánico no fue significativa en ninguna de las variables [Agorafobia: $F(1,217)=.498 ; \quad p=.481$. Depresión: $F(1,217)$ $=.161 ; p=.688$. Fobia Social: $F(1,217)=.615 ; p$ 434. Consumo de sustancias: $F(1,217)=.040$; $p=.843$ ].

En relación con una mayor presencia de la ideación / intento suicida en función de una mayor comorbilidad, se llevó a cabo un análisis de regresión múltiple, introduciendo como variables predictoras la puntuación en agorafobia, depresión, fobia social y consumo de sustancias. El modelo obtenido fue significativo $(F(4,216)=16,86 ; \quad p=.000)$, explicando casi un $25 \%$ de la varianza $\left(R^{2}=.22\right)$. En la Tabla 3 se resumen los coeficientes obtenidos. Como puede observarse, sólo el nivel de depresión alcanzó una elevada significación estadística. La puntuación en fobia social tuvo una participación apreciable, pero no llegó a ser significativa. Estos resultados indican que la adición de variables psicopatológicas no mejora la capacidad predictiva sobre el suicidio, más allá de la variable depresión. 
Finalmente, para conocer la capacidad predictiva de las variables tomadas en cuenta en este estudio sobre la ideación suicida (género, edad, nivel de estudios, estado civil, situación laboral y cronicidad presencia o no de pánico, depresión, ansiedad social, consumo de sustancias), se llevó a cabo una regresión logística, dividiendo de nuevo a la muestra entre personas sin ideación suicida y personas con ideación suicida. Los coeficientes obtenidos se resumen en la Tabla 4.

Tal y como se recoge en la Tabla 4, cuatro variables alcanzaron significación estadística. Claramente, el nivel de depresión se reveló como la variable de mayor peso (mayores niveles de depresión con la presencia de ideación suicida). Las otras tres variables fueron el nivel de estudios (con coeficiente negativo, indicando una mayor presencia de ideación suicida en personas en los niveles de estudios primarios y secundarios); la situación laboral, indicando una mayor presencia de ideación en las situaciones laborales más precarias (incapacitados y parados); y la edad, con una contribución negativa (mayor ideación a menor edad de los pacientes con agorafobia). La ecuación final resultante clasificó correctamente a un $83.3 \%$.

\section{Discusión}

La agorafobia conlleva una serie de limitaciones importantes para las personas que la sufren, afectando gravemente a su calidad de vida (interferencia con la vida cotidiana, movilidad limitada, restricción de la vida social/laboral, ...). Esta disminución en la calidad de vida se ve afectada porque con frecuencia la agorafobia cursa con otros problemas psicosociales (como ataques de pánico, otras fobias, depresión o conductas adictivas). El caso más grave sería la presencia de la ideación suicida.

Con este estudio se ha querido conocer, de forma general, la comorbilidad entre la agorafobia $\mathrm{y}$ otros trastornos frecuentes, especialmente en relación con el suicidio. Como resultado más relevante, la presencia de ideación suicida fue elevada, alcanzando a una de cada tres personas con agorafobia, y de ellas casi un 5\% indicaron que lo habían intentado o lo intentarían. Al mismo tiempo, los resultados muestran la existencia de una importante comorbilidad entre agorafobia y depresión, coincidente con otros estudios anteriores (i.e., Brown et al., 2001; Wittchen, Nocon, \& Beesdo, 2008): nueve de cada diez pacientes mostraron niveles de depresión clínicamente relevantes. También se pudo constatar la comorbilidad con la fobia / ansiedad social (Magee et al.,1996). Sin embargo, la relación con conductas adictivas no pudo ser establecida: gran parte de la muestra no consumía alcohol, tabaco, cannabis, estimulantes $\mathrm{u}$ otras drogas, que contradice hallazgos precedentes (Huang et al., 2010). Puede ocurrir en este último caso que, dado que la información sobre el consumo de sustancias era autoinformado por el paciente durante una entrevista, la información pudiera estar afectada por la deseabilidad social.

También se ha podido verificar la vinculación estrecha entre depresión y la presencia de la ideación suicida: se ha podido constatar que las personas con agorafobia y comorbilidad con depresión presentaban un mayor riesgo de ideación e intentos de suicidio, coincidiendo de nuevo con estudios anteriores (Bierut, Heath, \& Bucholz, 1999; Sareen et al., 2005; Starcevic et al., 1999). En este sentido hay que destacar que la mayor gravedad de la agorafobia (una mayor puntuación en el inventario AGF) no se relacionó con una mayor ideación suicida. Lo que sí parece implicar la aparición de esa ideación es la presencia de la depresión. Por otro lado, no ha podido constatarse que la presencia de crisis de pánico o la comorbilidad con la ansiedad social afectara a una mayor presencia de pensamientos suicidas, lo que se contradice con la revisión de Kilbane et al., (2009) y está más acorde con el meta-análisis de Bentley et al. (2016). Tampoco se pudo confirmar que el consumo de sustancias / consumo de alcohol estuviera asociado a una mayor vinculación entre la agorafobia y el suicidio, como se había informado previamente (Huang et al., 2010). La idea, por tanto, de que a mayor comorbilidad mayor ideación suicida no pudo constatarse en nuestro estudio. Este resultado entronca con un paradigma de investigación muy sólido que relaciona estrechamente depresión y suicidio (Beck, Steer, Kovacs, \& Garrison, 1985), con la desesperanza como mejor predictor (Hawton, Combabella, 
Haw, \& Saunders, 2013). Asímismo está directamente relacionado con las señales de riesgo suicida, especialmente la vinculada al comportamiento emocional (cambios de humor, irritabilidad, ira...) y aislamiento / evitación depresiva (Brás, Carmo, \& Jesus, 2017).

Cuando se analizan las contribuciones específicas de variables psicopatológicas y sociodemográficas (regresión logística), claramente es el nivel de depresión el que mejor se asocia con esa ideación suicida. Después aparecen asociadas variables de carácter sociodemográfico como una mayor precariedad laboral, menor nivel de estudios y la edad. En el caso de la edad la relación es negativa, (similar a los datos encontrados por Huang et al., 2010), indicando una mayor presencia de ideación suicida en pacientes más jóvenes. Este dato se corrobora con la ausencia de participación del nivel de cronicidad de la agorafobia, no pudiéndose establecer una mayor presencia de ideación suicida en personas con mayor tiempo con el trastorno. Al contrario, la ideación aparece tempranamente y luego parece diluirse con el paso del tiempo. Este es un resultado aparentemente contraintuitivo, porque sería esperable que, a medida que el paciente observe que su problema se agrava y cronifica, la idea del suicidio como una solución empezaría a aparecer. Por el contrario, lo que los datos parecen indicar es que, después del impacto inicial del problema (y su interferencia con la vida cotidiana), el paciente parece 'adaptarse' al problema y esos pensamientos auto-líticos se van disipando. También es posible que, con el paso de los años, algunos factores protectores, como el bienestar psicológico (Alarcón-Vásquez et al., 2019), puedan jugar un mayor papel mediador.

Las implicaciones prácticas de estos resultados pueden tener relación con la vinculación entre un conjunto de síntomas directamente asociados con la depresión (sentimientos de desamparo, comportamiento emocional negativo y evitación depresiva) y el riesgo suicida. Esto es especialmente relevante para las personas que sufren agorafobia, por su vinculación tanto a la depresión como a la ideación suicida. Los resultados de los tratamientos cognitivo-conductuales sobre la agorafobia han mostrado cómo la disminución de los síntomas agorafóbicos va asociada con una disminución en los síntomas depresivos (Peñate et al., 2017; Pitti et al., 2015), lo que podría implicar una disminución del riesgo suicida. $\mathrm{Si}$ la depresión fuera una consecuencia de la agorafobia y la ideación suicida estuviera directamente relacionada con la depresión, entonces, paradójicamente, la presencia de la agorafobia podría suponer un buen pronóstico para el riesgo suicida, en la medida en que un buen abordaje terapéutico de la misma supondría una disminución de los niveles de depresión y dicho riesgo. No ocurre los mismo cuando el riesgo suicida está asociado directamente con la desesperanza, donde la tendencia auto-lítica es más compleja de modificar (Cuijpers et al., 2013; Teismann, Forkmann, Rath, Glaesmer, \& Margraf, 2016).

Este trabajo presenta varias limitaciones. A pesar de que se contó con una muestra apreciable de pacientes, esta fue una muestra incidental, lo cual indica que no fue seleccionada aleatoriamente, con las implicaciones que esto pudo suponer para la generalización de los resultados. La medición del consumo de sustancias se ha hecho de manera generalista y no se tomó en cuenta posibles problemas de deseabilidad social. Frecuentemente, hemos reducido los análisis a la ideación suicida, sin embargo, existe una clara diferenciación entre ideación e intento suicida. Con muestras mayores se podría analizar esta diferenciación, que no ha podido hacerse en este estudio. Además, este es un estudio retrospectivo, un estudio longitudinal podría ofrecer resultados más precisos.

En definitiva, este trabajo muestra como la agorafobia suele cursar con otros problemas comórbidos, donde la ideación suicida está muy presente. Desde el punto de vista de los factores predictores del riesgo suicida, destaca el papel del nivel de depresión y determinados factores sociodemográficos. La comorbilidad con la ansiedad social, la presencia de ataques de pánico y el consumo de sustancias no se relacionó con una mayor presencia de ideación suicida. Tampoco pudo verificarse que a mayor comorbilidad mayor incremento en la capacidad predictiva de la ideación suicida. 


\section{Referencias}

Abreu, L. N., Oquendo, M. A., Galfavy, H., Burke, A., Grunebaum, M. F., Sher, L., ... \& Lafer, B. (2018). Are comorbid anxiety disorders a risk factor for suicide attempts in patients with mood disorders? A two-year prospective study. European Psychiatry, 47(1), 19-24. doi:10.1016/j.eurpsy.2017.09.005

Alarcón-Vásquez, Y., Bahamón, M. J., TrejosHerrera, A. M., González-Gutiérrez, O., Rubio-Castro, R., Hernández-Cervantes, Q., \& Gómez-Maqueo, E. L. (2019). Análisis de las propiedades psicométricas del inventario de riesgo suicida (IRISA) para adolescentes colombianos. Revista Iberoamericana de Diagnóstico y Evaluación - e Avaliação Psicológica, 2(51), 87-98. doi:10.21865/RIDEP51.2.07

American Psychiatric Association (2013). Diagnostic and statistical manual of mental disorders: DSM-V (Fifth Edition). Washington D.C.: Author.

An, J. H., Lee, K. E., Lee, H. C., Kim, H. S., Jun, J. Y., Chang, H. I., ... \& Hong, J. P. (2018). Prevalence and correlates of suicidal thoughts and behaviors among north korean defectors. Psychiatry investigation, 15(5), 445-451. doi:10.30773/pi.2017.11.08.3

Ashrafioun, L., Pigeon, W. R., Conner, K. R., Leong, S. H., \& Oslin, D. W. (2016). Prevalence and correlates of suicidal ideation and suicide attempts among veterans in primary care referred for a mental health evaluation. Journal of Affective Disorders, 189(1), 344-350. doi:10.1016/j.jad.2015.09.014

Baca, E., \& Aroca, F. (2014). Factores de riesgo de la conducta suicida asociados a trastornos depresivos y ansiedad. Salud Mental, 37(5), 373-380.

Bados, A. (2006). Tratando el pánico y agorafobia. Madrid: Pirámide.

Beck, A. T., Steer, R. A., Kovacs, M., \& Garrison, B. (1985). Hopelessness and eventual suicide: A 10-year prospective study of patients hospitalized with suicidal ideation. The American Journal of Psychiatry, 142(5), 559563. doi:10.1176/ajp.142.5.559
Beck, A., Steer, R., \& Brown, G. (1996). Manual for the Beck Depression Inventory-II. San Antonio, Texas: Psychological Corporation.

Bentley, K. H., Franklin, J. C., Ribeiro, J. D., Kleiman, E. M., Fox, K. R., \& Nock, M. K. (2016). Anxiety and its disorders as risk factors for suicidal thoughts and behaviors: A meta-analytic review. Clinical Psychology Review, 43(2), 30-46.

doi:10.1016/j.cpr.2015.11.008

Bierut, L., Heath, A., \& Bucholz, K. (1999). Major depressive disorder in a community based twin sample are there different genetic and environmental contributions for men and women? Archives of General Psychiatry, 56(6), 557-563. doi:10.1001/archpsyc.56.6.557

Brás, M., Carmo, C., \& Jesus, S. N. (2017). Estudo das propriedades psicométricas do inventário de reconhecimento de sinais de alerta para atos suicidas. Revista Iberoamericana de Diagnóstico y Evaluación - e Avaliação Psicológica, 43(1), 89-105. doi:10.21865/RIDEP43

Brown, T. A., Campbell, L. A., Lehman, C. L., Grisham, J. R., \& Mancill, R. B. (2001). Current and lifetime comorbidity of the DSMIV anxiety and mood disorders in a large clinical sample. Journal of Abnormal Psychology, 110(4), 585-599. doi:10.1037/0021-843X.110.4.585

Cuijpers, P., de Beurs, D. P., van Spijker, B. A., Berking, M., Andersson, G., \& Kerkhof, A. J. (2013). The effects of psychotherapy for adult depression on suicidality and hopelessness: A systematic review and meta-analysis. Journal of Affective Disorder, 144(3), 183-190. doi:10.1016/j.jad.2012.06.025

Delgado, B., Inglés, C. J., Aparisi, D., GarcíaFernández, J. M., \& Martínez-Monteagudo, M. C. (2018). Relación entre la ansiedad social y las dimensiones de la personalidad en adolescentes españoles. Revista Iberoamericana de Diagnóstico y Evaluación - e Avaliação Psicológica, 46(1), 81-92. doi:10.21865/RIDEP46.1.06

Echeburúa, E., Corral, P., García, E., Páez, D., \& Borda, M. (1992). Un nuevo Inventario de Agorafobia (IA). Análisis y Modificación de Conducta, 18(57), 101-123. 
Gómez-Tabares, A. S., Núñez, C., AgudeloOsorio, M. P., \& Grisales-Aguirre, A. M. (2020). Riesgo e ideación suicida y su relación con la impulsividad y la depresión en adolescentes escolares. Revista Iberoamericana de Diagnóstico y Evaluación - e Avaliação Psicológica, 1(54), 147-163. doi:10.21865/RIDEP54.1.12

Grant, B. F., Dawson, D. A., Stinson, F. S., Chou, P. S., Kay, W., \& Pickering, R. (2003). The alcohol use disorder and associated disabilities interview schedule-IV (AUDADIS-IV): Reliability of alcohol consumption, tobacco use, family history of depression and psychiatric diagnostic modules in a general population sample. Drug and Alcohol Dependence, 71(1), 7-16. doi:10.1016/S0376-8716(03)00070-X

Hawton, K., Combabella, C. C., Haw, C., \& Saunders, K. (2013). Risk factors for suicide in individuals with depression: A systematic review. Journal of Affective Disorders, 147(13), 17-28. doi:10.1016/j.jad.2013.01.004

Heimberg, R. G., Horner, K. J., Juster, H. R., Safren, S. A., Brown, E. J., Schneier, F.R.,, \& Liebowitz, M. R. (1999). Psychometric properties of the Liebowitz Social Anxiety Scale. Psychological Medicine, 29(1), 199212. doi:10.1017/S0033291798007879

Huang, M. F., Yen, C. F., \& Lung, F. W. (2010). Moderators and mediators among panic, agoraphobia symptoms, and suicidal ideation in patients with panic disorder. Comprehensive Psychiatry, 51, 243-249. doi:10.1016/j.comppsych.2009.07.005

Inoue, K., Kaiya, H., Hara, N., \& Okazaki, Y. (2016). A discussion of various aspects of panic disorder depending on presence or absence of agoraphobia. Comprehensive Psychiatry, 69, 132-135.

doi:10.1016/j.comppsych.2016.05.014

Kessler, R., \& Üstün, T. (2004). The World Mental Health (WMH) Survey Initiave Version of the World Health Organization, Composite International Diagnostic Interview (CIDI). International Journal of Methods in Psychiatric Research, 13(2), 93-121. doi:10.1002/mpr.168

Kessler, R., Petukhova, M., Sampson, A., Zaslavky, A., \& Wittchen, H. (2012). Twelve month and lifetime prevalence and lifetime morbid risk of anxiety and mood disorders in the United States. International Journal of Methods in Psychiatric Research, 21(3), 169184. doi:10.1002/mpr.1359

Kilbane, E. J., Gokbayrak, N. S., Galynker, I., Cohen, L., \& Tross, S. (2009). A review of panic and suicide in bipolar disorder: Does comorbidity increase risk? Journal of Affective Disorders, 115(1-2), 1-10. doi:10.1016/j.jad.2008.09.014

Liebowitz, M. R. (1987). Social phobia. En D.F. Klein (Ed.). Modern Problems of Pharmacopsychiatry, Vol. 22 (pp. 141-173). Basel: Karger. doi:10.1159/000414022

Magee, W. J., Eaton, W. W., Wittchen, H. U., McGonagle, K. A., \& Kessler, R. (1996). Agoraphobia, simple phobia, and social phobia in the National Comorbidity Survey. Archives of General Psychiatry, 53(2), 159-168. doi:10.1001/archpsyc.1996.01830020077009

Miché, M., Hofer, P. D., Voss, C., Meyer, A. H., Gloster, A. T., Beesdo-Baum, K., \& Lieb, R. (2017). Mental disorders and the risk for the subsequent first suicide attempt: Results of a community study on adolescents and young adults. European Child \& Adolescent Psychiatry, 27(7), 839-848. doi:10.1007/s00787-017-1060-5

Norton, P., Temple, S., \& Pettit, J. (2008). Suicidal ideation and anxiety disorders: Elevated risk or artifact of comorbid depression? Journal of Behavior Therapy and Experimental Psychiatry, 39(4), 515-525. doi:10.1016/j.jbtep.2007.10.010

Orri, M., Galera, C., Turecki, G., Forte, A., Renaud, J., Boivin, M., ... \& Geoffroy, M-C. (2018). Association of childhood irritability and depressive/anxious mood profiles with adolescent suicidal ideation and attempts. JAMA Psychiatry, 75(5), 465-473. doi:10.1001/jamapsychiatry.2018.0174

Peñate, W., Roca-Sánchez, M. J., Pitti, C. T., Villaverde, M. L., Bethencourt, J. M., Álvarez-Pérez, Y... \& Gracia, R. (2017). Efficacy of an Internet-based psychological treatment for agoraphobia with minimal therapist contact. Revista Iberoamericana de Psicología y Salud, 8(2), 85-95. doi:10.23923/j.rips.2017.08.008 
Pitti, C. T., Peñate, W., de la Fuente, J., Bethencourt, J. M., Roca-Sánchez, M. J., Acosta. L., ..., \& Gracia, R. (2015). The combined use of virtual reality exposure in the treatment of agoraphobia. Actas Españolas de Psiquiatría, 43(4), 133-141. PMID:26150057

Sánchez-Álvarez, N., de la Torre-López, J., \& Pérez-Costillas, L. (2018). Construcción y evaluación psicométrica de una escala breve de vulnerabilidad suicida. Revista Iberoamericana de Diagnóstico y Evaluación - e Avaliação Psicológic, 4(49) 23-35.

doi:10.21865/RIDEP49.4.02

Sareen, J., Cox, B., \& Afifi, T. (2005). Anxiety disorders and risk for suicidal ideation and suicide attempts: A population based longitudinal study of adults. Archives of General Psychiatry, 62(2), 1249-1257.

doi:10.1001/archpsyc.62.11.1249

Starcevic, V., Bogojevic, G., Marinkovic, J., \& Kelin, K. (1999). Axis I and Axis II comorbidity in panic/agoraphobic patients with and without suicidal ideation. Psychiatry Research, 88(2), 153-161. doi:10.1016/S0165-1781(99)00078-5

Teismann, T., Forkmann, T., Rath, D., Glaesmer, H., \& Margraf, J. (2016). Perceived burdensomeness and suicide ideation in adult outpatients receiving exposure therapy for anxiety disorders. Behaviour Research and Therapy, 85(1), 1-5. doi:10.1016/j.brat.2016.07.011

Teismann, T., Lukaschek, K., Hiller, T. S., Breitbart, J., Brettschneider, C., Schumacher, U., ... \& Paradies, J. (2018). Suicidal ideation in primary care patients suffering from panic disorder with or without agoraphobia. $B M C$ Psychiatry 18, 305.

doi:10.1186/s12888-018-1894-5

WHO (1992). ICD-10: International Statistical Classification of Diseases and Related Health Problems. Geneve: Autor.

Wittchen, H., Nocon, A., \& Beesdo, K. (2008). Agoraphobia and panic. Psychotherapy and Psychosomatics, 77(1), 147-157.

doi:10.1159/000116608 